MATHEMATICS OF COMPUTATION

Volume 76, Number 257, January 2007, Pages 287-298

S $0025-5718(06) 01892-8$

Article electronically published on August 31, 2006

\title{
CONVERGENCE PROPERTIES OF PRECONDITIONED HERMITIAN AND SKEW-HERMITIAN SPLITTING METHODS FOR NON-HERMITIAN POSITIVE SEMIDEFINITE MATRICES
}

\author{
ZHONG-ZHI BAI, GENE H. GOLUB, AND CHI-KWONG LI
}

\begin{abstract}
For the non-Hermitian and positive semidefinite systems of linear equations, we derive necessary and sufficient conditions for guaranteeing the unconditional convergence of the preconditioned Hermitian and skewHermitian splitting iteration methods. We then apply these results to block tridiagonal linear systems in order to obtain convergence conditions for the corresponding block variants of the preconditioned Hermitian and skew-Hermitian splitting iteration methods.
\end{abstract}

\section{INTRODUCTION}

We consider the iterative solution of a large sparse non-Hermitian system of linear equations

$$
A x=b, A \in \mathbf{C}^{n \times n} \text { nonsingular, } A \neq A^{*} \text {, and } x, b \in \mathbf{C}^{n},
$$

where $A^{*}$ denotes the conjugate transpose of the complex matrix $A$.

Based on the Hermitian and skew-Hermitian (HS) splitting

$$
A=\mathcal{H}(A)+\mathcal{S}(A), \text { with } \mathcal{H}(A)=\frac{1}{2}\left(A+A^{*}\right) \text { and } \mathcal{S}(A)=\frac{1}{2}\left(A-A^{*}\right),
$$

Bai, Golub and Ng recently established a class of Hermitian and skew-Hermitian splitting (HSS) iteration methods in [3] for solving the non-Hermitian system of linear equations (1.1).

When the coefficient matrix $A \in \mathbf{C}^{n \times n}$ is positive definite, i.e., its Hermitian part $\mathcal{H}(A) \in \mathbf{C}^{n \times n}$ is Hermitian positive definite, they proved in [3] that the HSS iteration converges unconditionally to the exact solution of the system of linear equations (1.1), with the bound on the rate of convergence about the same as that

Received by the editor February 11, 2005.

2000 Mathematics Subject Classification. Primary 65F10, 65F50.

Key words and phrases. Non-Hermitian matrix, positive semidefinite matrix, Hermitian and skew-Hermitian splitting, splitting iteration method, convergence.

The work of the first author was supported by The Special Funds For Major State Basic Research Projects (No. G1999032803), The National Basic Research Program (No. 2005CB321702), The China NNSF Outstanding Young Scientist Foundation (No. 10525102) and The National Natural Science Foundation (No. 10471146), P.R. China, and The 2004 Ky and Yu-Fen Fan Fund Travel Grant of American Mathematical Society.

The work of the second author was in part supported by the Department of Energy: DE-FC02$01 \mathrm{ER} 41177$.

The research of the third author was partially supported by an NSF grant. 
of the conjugate gradient method when applied to the Hermitian matrix $\mathcal{H}(A)$. Moreover, the upper bound of the contraction factor is dependent on the spectrum of the Hermitian part $\mathcal{H}(A)$, but is independent of the spectrum of the skew-Hermitian part $\mathcal{S}(A)$ as well as the eigenvalues of the matrices $\mathcal{H}(A), \mathcal{S}(A)$, and $A$. Numerical experiments have shown that the HSS iteration method is very efficient and robust for solving non-Hermitian positive definite linear systems; see 3 .

When the coefficient matrix $A \in \mathbf{C}^{n \times n}$ has the two-by-two block structure

$$
A=\left(\begin{array}{cc}
B & E \\
-E^{*} & C
\end{array}\right)
$$

with $B \in \mathbf{C}^{p \times p}$ being positive definite (i.e., $\mathcal{H}(B)$ is Hermitian positive definite), $C \in \mathbf{C}^{q \times q}$ being Hermitian positive semidefinite, and $E \in \mathbf{C}^{p \times q}$ being of full column rank, Benzi, and Golub further proved in [11] that the HSS iteration method for the corresponding saddle-point problem

$$
A x \equiv\left(\begin{array}{cc}
B & E \\
-E^{*} & C
\end{array}\right)\left(\begin{array}{l}
y \\
z
\end{array}\right)=\left(\begin{array}{c}
f \\
g
\end{array}\right) \equiv b
$$

also converges unconditionally to its exact solution. Note that the matrix $A$ is now only positive semidefinite with some special structure, namely, its Hermitian part

$$
\mathcal{H}(A)=\left(\begin{array}{cc}
\mathcal{H}(B) & 0 \\
0 & C
\end{array}\right) \in \mathbf{C}^{n \times n}
$$

is such that $\mathcal{H}(B)$ is positive definite and $C$ is Hermitian positive semidefinite.

In this paper, we give a necessary and sufficient condition for an arbitrary nonHermitian positive semidefinite linear system so that the preconditioned Hermitian and skew-Hermitian splitting (PHSS) iteration method will lead to an unconditionally convergent iteration sequence. This result is further specialized to linear systems of a block tridiagonal form to obtain unconditional convergence conditions for the corresponding block PHSS (BPHSS) iteration method.

\section{The PRECONDITIONED HSS METHOD}

Instead of applying the HSS iteration technique directly to the system of linear equations (1.1), we may apply it to the systematically preconditioned linear system

$$
\widehat{A} \widehat{x}=\widehat{b}, \text { with } \widehat{A}=R^{-*} A R^{-1}, \widehat{x}=R x \text {, and } \widehat{b}=R^{-*} b,
$$

where $R \in \mathbf{C}^{n \times n}$ is a prescribed nonsingular matrix and $R^{-*}=\left(R^{-1}\right)^{*}=\left(R^{*}\right)^{-1}$. Let $P=R^{*} R$. Then $P \in \mathbf{C}^{n \times n}$ is a Hermitian positive definite matrix. This leads to the preconditioned Hermitian and skew-Hermitian splitting (PHSS) iteration method as follows. See also [2, 3, 4] and [11, 12.

The PHSS iteration method. Let $P \in \mathbf{C}^{n \times n}$ be a prescribed Hermitian positive definite matrix. Given an initial guess $x^{(0)} \in \mathbf{C}^{n}$, compute $x^{(k)}$ for $k=0,1,2, \ldots$ using the following iteration scheme until $\left\{x^{(k)}\right\}$ satisfies the stopping criterion:

$$
\left\{\begin{aligned}
(\alpha P+\mathcal{H}(A)) x^{\left(k+\frac{1}{2}\right)} & =(\alpha P-\mathcal{S}(A)) x^{(k)}+b, \\
(\alpha P+\mathcal{S}(A)) x^{(k+1)} & =(\alpha P-\mathcal{H}(A)) x^{\left(k+\frac{1}{2}\right)}+b,
\end{aligned}\right.
$$

where $\alpha$ is a given positive constant.

Clearly, when $P=I$, the identity matrix, the PHSS iteration method reduces to the HSS iteration method studied in Bai, Golub, and $\mathrm{Ng}$ in 3 . When $P \neq I$, we can 
suitably choose $P$ and $\alpha$ such that the induced PHSS iteration method possesses fast convergence and high computing efficiency. In addition, the Hermitian positive definite matrix $P$ and the positive constant $\alpha$ should be judiciously selected so that the two subsystems of linear equations with the coefficient matrices $\alpha P+\mathcal{H}(A)$ and $\alpha P+\mathcal{S}(A)$ can be solved economically and rapidly.

In matrix-vector form, the above PHSS iteration method can be rewritten as

$$
x^{(k+1)}=\mathcal{L}(\alpha, P) x^{(k)}+\mathcal{G}(\alpha, P) b, \quad k=0,1,2, \ldots,
$$

where

$$
\mathcal{L}(\alpha, P)=(\alpha P+\mathcal{S}(A))^{-1}(\alpha P-\mathcal{H}(A))(\alpha P+\mathcal{H}(A))^{-1}(\alpha P-\mathcal{S}(A))
$$

and

$$
\mathcal{G}(\alpha, P)=2 \alpha(\alpha P+\mathcal{S}(A))^{-1}(\alpha P+\mathcal{H}(A))^{-1} .
$$

Here, $\mathcal{L}(\alpha, P)$ is the iteration matrix of the PHSS iteration method. In fact, (2.2) may also result from the splitting

$$
A=\mathcal{M}(\alpha, P)-\mathcal{N}(\alpha, P)
$$

of the coefficient matrix $A$, with

$$
\left\{\begin{array}{l}
\mathcal{M}(\alpha, P)=\frac{1}{2 \alpha}(\alpha P+\mathcal{H}(A))(\alpha P+\mathcal{S}(A)) \\
\mathcal{N}(\alpha, P)=\frac{1}{2 \alpha}(\alpha P-\mathcal{H}(A))(\alpha P-\mathcal{S}(A))
\end{array}\right.
$$

Therefore, the PHSS iteration method can naturally induce a preconditioner $\mathcal{M}(\alpha, P)$ to the matrix $A$. This preconditioner is called as the PHSS preconditioner. See [2, 4, 11, 12].

When $A \in \mathbf{C}^{n \times n}$ is a positive definite matrix, duplicating the proofs of Theorem 2.2 and Corollary 2.3 in 3 allows one to establish the following convergence theorem for the PHSS iteration method. In the sequel, $\operatorname{sp}(X)$ represents the spectrum of the square matrix $X$.

Theorem 2.1. Let $A \in \mathbf{C}^{n \times n}$ be a positive definite matrix, let $\mathcal{H}(A)=\frac{1}{2}\left(A+A^{*}\right)$ and $\mathcal{S}(A)=\frac{1}{2}\left(A-A^{*}\right)$ be its Hermitian and skew-Hermitian parts, respectively, and let $\alpha$ be a positive constant. Let $P \in \mathbf{C}^{n \times n}$ be a Hermitian positive definite matrix. Then the spectral radius $\rho(\mathcal{L}(\alpha, P))$ of the iteration matrix $\mathcal{L}(\alpha, P)$ of the PHSS iteration is bounded by

$$
\sigma(\alpha, P)=\max _{\lambda_{j} \in \operatorname{sp}\left(P^{-1} \mathcal{H}(A)\right)} \frac{\left|\alpha-\lambda_{j}\right|}{\left|\alpha+\lambda_{j}\right|} .
$$

Consequently, we have

$$
\rho(\mathcal{L}(\alpha, P)) \leq \sigma(\alpha, P)<1, \quad \forall \alpha>0,
$$

i.e., the PHSS iteration unconditionally converges to the exact solution of the system of linear equations (1.1).

Moreover, if $\gamma_{\min }$ and $\gamma_{\max }$ are the lower and the upper bounds of the eigenvalues of the matrix $P^{-1} \mathcal{H}(A)$, respectively, then

$$
\tilde{\alpha}:=\arg \min _{\alpha}\left\{\max _{\gamma_{\min } \leq \lambda \leq \gamma_{\max }}\left|\frac{\alpha-\lambda}{\alpha+\lambda}\right|\right\}=\sqrt{\gamma_{\min } \gamma_{\max }}
$$

and

$$
\sigma(\tilde{\alpha}, P)=\frac{\sqrt{\gamma_{\max }}-\sqrt{\gamma_{\min }}}{\sqrt{\gamma_{\max }}+\sqrt{\gamma_{\min }}}=\frac{\sqrt{\kappa\left(P^{-1} \mathcal{H}(A)\right)}-1}{\sqrt{\kappa\left(P^{-1} \mathcal{H}(A)\right)}+1},
$$

where $\kappa\left(P^{-1} \mathcal{H}(A)\right)$ is the spectral condition number of the matrix $P^{-1} \mathcal{H}(A)$. 
From Theorem 2.1 we see that the Hermitian positive definite matrix $P \in \mathbf{C}^{n \times n}$ should be chosen such that it is at least a good approximate to the matrix $\mathcal{H}(A)$. In this situation, $\kappa\left(P^{-1} \mathcal{H}(A)\right)$ may be reasonably small so that the PHSS iteration method may achieve fast convergence. On the other hand, since we often have to solve the two half-iterates $x^{\left(k+\frac{1}{2}\right)}$ and $x^{(k+1)}$ inexactly by some iteration schemes, $P$ and $\alpha$ should be chosen such that both matrices $\alpha P+\mathcal{H}(A)$ and $\alpha P+\mathcal{S}(A)$ are well conditioned and economically invertible. Hence, in a practical computation, it is a crucial but difficult problem to determine a good preconditioner $P$ and choose an optimal iteration parameter $\alpha$. For some discussions on this aspect, we refer the readers to [2, 4, 11, 12.

\section{Convergence theorems}

In this section, we study the convergence properties of the PHSS iteration method when the coefficient matrix $A \in \mathbf{C}^{n \times n}$ is positive semidefinite. To this end, we call an eigenvalue $\lambda$ of a matrix $W \in \mathbf{C}^{n \times n}$ a reducing eigenvalue if $W x=\lambda x$ and $W^{*} x=\lambda^{*} x$. Equivalently, $W$ is unitarily similar to $[\lambda] \oplus W_{0}$, where $W_{0} \in$ $\mathbf{C}^{(n-1) \times(n-1)}$.

The following theorem describes the convergence property of the PHSS iteration method when the coefficient matrix $A \in \mathbf{C}^{n \times n}$ is positive semidefinite.

Theorem 3.1. Let $A \in \mathbf{C}^{n \times n}$ be a positive semidefinite matrix, let $\mathcal{H}(A)=\frac{1}{2}(A+$ $\left.A^{*}\right)$ and $\mathcal{S}(A)=\frac{1}{2}\left(A-A^{*}\right)$ be its Hermitian and skew-Hermitian parts, respectively, and let $\alpha$ be a positive constant. Let $P \in \mathbf{C}^{n \times n}$ be a Hermitian positive definite matrix. Then the spectral radius $\rho(\mathcal{L}(\alpha, P))$ of the iteration matrix $\mathcal{L}(\alpha, P)$ of the PHSS iteration is bounded by 1 , i.e.,

$$
\rho(\mathcal{L}(\alpha, P)) \leq 1, \quad \forall \alpha>0 .
$$

The inequality becomes an equality if and only if the matrix $\widehat{A}:=R^{-*} A R^{-1}$ has a (reducing) eigenvalue of the form $i \xi$ with $\xi \in \mathbf{R}$ and $i$ the imaginary unit, i.e., the null space of $\mathcal{H}(\widehat{A})$ contains an eigenvector of $\mathcal{S}(\widehat{A})$. Here, $P=R^{*} R$ and $R \in \mathbf{C}^{n \times n}$ is a prescribed nonsingular matrix.

Proof. Evidently, we only need to consider the case when $P=I$, as otherwise, we can turn to the preconditioned linear system (2.1) instead. Denote by

$$
\mathcal{L}(\alpha):=\mathcal{L}(\alpha, I)=(\alpha I+\mathcal{S}(A))^{-1}(\alpha I-\mathcal{H}(A))(\alpha I+\mathcal{H}(A))^{-1}(\alpha I-\mathcal{S}(A)),
$$

which is similar to the matrix

$$
\overline{\mathcal{L}}(\alpha):=(\alpha I+\mathcal{H}(A))^{-1}(\alpha I-\mathcal{H}(A))(\alpha I+\mathcal{S}(A))^{-1}(\alpha I-\mathcal{S}(A)) .
$$

Therefore, we only need to investigate the property of the eigenvalues of $\overline{\mathcal{L}}(\alpha)$.

Suppose that $\mathcal{H}(A)$ has eigenvalues

$$
\mu_{1} \geq \cdots \geq \mu_{r}>0=\mu_{r+1}=\cdots=\mu_{n}=0 .
$$

Then $(\alpha I+\mathcal{H}(A))^{-1}(\alpha I-\mathcal{H}(A))$ is Hermitian and has eigenvalues

$$
\nu_{j}=\left(\alpha-\mu_{j}\right) /\left(\alpha+\mu_{j}\right), \quad j=1,2, \ldots, n,
$$

so that

$$
-1<\nu_{1} \leq \cdots \leq \nu_{r}<1=\nu_{r+1}=\cdots=\nu_{n}
$$


Hence $\overline{\mathcal{L}}(\alpha)$ has singular values

$$
\left|\nu_{j}\right| \leq 1, \quad j=1,2, \ldots, n .
$$

Consequently,

$$
\rho(\overline{\mathcal{L}}(\alpha)) \leq\|\overline{\mathcal{L}}(\alpha)\|=1, \quad \forall \alpha>0 .
$$

Suppose that $A$ has an eigenvalue of the form $i \xi$ with $\xi \in \mathbf{R}$ corresponding to a unit eigenvector $v$. We show that $i \xi$ is in fact a reducing eigenvalue of $A$. To see this, let $V$ be a unitary matrix such that $V^{*} A V$ is in lower triangular form with $i \xi$ in the $(1,1)$ entry, and $w$ as the first column. Then

$$
\mathcal{H}\left(V^{*} A V\right)=V^{*} \mathcal{H}(A) V
$$

is positive semidefinite with 0 in the $(1,1)$ entry and $\frac{1}{2} w$ as the first column. It follows that $w=0$ and $U^{*} A U=[i \xi] \oplus A_{0}$ for some $A_{0} \in \mathbf{C}^{(n-1) \times(n-1)}$, i.e., $i \xi$ is a reducing eigenvalue of $A$. Now,

$$
(\alpha I+\mathcal{H}(A))^{-1}(\alpha I-\mathcal{H}(A))(\alpha I+\mathcal{S}(A))^{-1}(\alpha I-\mathcal{S}(A)) v=\lambda v
$$

with $\lambda=(\alpha-i \xi) /(\alpha+i \xi)$ such that $|\lambda|=1$.

Conversely, if $\overline{\mathcal{L}}(\alpha)$ has an eigenvalue $\lambda$ of modulus 1 , then $\lambda$ is an eigenvalue of the matrix

$$
(\alpha I+\mathcal{S}(A))^{-1}(\alpha I-\mathcal{S}(A))(\alpha I+\mathcal{H}(A))^{-1}(\alpha I-\mathcal{H}(A)) .
$$

Thus, there is a unit vector $v \in \mathbf{C}^{n}$ such that

$$
(\alpha I+\mathcal{S}(A))^{-1}(\alpha I-\mathcal{S}(A))(\alpha I+\mathcal{H}(A))^{-1}(\alpha I-\mathcal{H}(A)) v=\lambda v .
$$

Since $|\lambda|=1$ and $(\alpha I+\mathcal{S}(A))^{-1}(\alpha I-\mathcal{S}(A))$ is unitary, we see that

$$
\left\|(\alpha I+\mathcal{H}(A))^{-1}(\alpha I-\mathcal{H}(A)) v\right\|=\|v\| .
$$

Suppose that $\left\{\mathrm{x}_{1}, \mathrm{x}_{2}, \ldots, \mathrm{x}_{n}\right\}$ is an orthonormal basis for $\mathbf{C}^{n}$ consisting of eigenvectors of $\mathcal{H}(A)$ such that $\mathcal{H}(A) \mathrm{x}_{j}=\mu_{j} \mathrm{x}_{j}$ for $j=1,2, \ldots, n$. Let $v=\sum_{j=1}^{n} \theta_{j} \mathrm{x}_{j}$ with $\theta_{j} \in \mathbf{C}$. Then

$$
1=\sum_{j=1}^{n}\left|\theta_{j}\right|^{2}=\|v\|^{2}=\left\|(\alpha I+\mathcal{H}(A))^{-1}(\alpha I-\mathcal{H}(A)) v\right\|^{2}=\sum_{j=1}^{n}\left|\theta_{j}\right|^{2} \nu_{j}^{2} .
$$

By (3.1), we know that $\theta_{j}=0$ for $j=1,2, \ldots, r$. It follows that $v=\sum_{j=r+1}^{n} \theta_{j} \mathrm{x}_{j}$ and

$$
\mathcal{H}(A) v=\sum_{j=r+1}^{n} \theta_{j} \mathcal{H}(A) \mathrm{x}_{j}=\sum_{j=r+1}^{n} \theta_{j} \mu_{j} \mathrm{x}_{j}=0 .
$$

Furthermore,

$$
\begin{aligned}
\lambda v & =(\alpha I+\mathcal{S}(A))^{-1}(\alpha I-\mathcal{S}(A))(\alpha I+\mathcal{H}(A))^{-1}(\alpha I-\mathcal{H}(A)) v \\
& =(\alpha I+\mathcal{S}(A))^{-1}(\alpha I-\mathcal{S}(A)) v .
\end{aligned}
$$

Thus, $v$ is an eigenvector of $(\alpha I+\mathcal{S}(A))^{-1}(\alpha I-\mathcal{S}(A))$, and hence $v$ is an eigenvector of $\mathcal{S}(A)$ such that $\mathcal{S}(A) v=i \xi v$ with $\xi \in \mathbf{R}$ satisfying $\lambda=(\alpha-i \xi) /(\alpha+i \xi)$. As a result, $A v=i \xi v$ and $A^{*} v=-i \xi v$. So, $i \xi$ is a reducing eigenvalue of $A$.

Corollary 3.2. Suppose that $A \in \mathbf{C}^{n \times n}$ satisfies the hypothesis of Theorem 3.1. If $\rho(\mathcal{L}(\alpha, P))<1$, then $A$ is nonsingular. 
The contra-positive of the above corollary asserts that if a matrix $A$ satisfying the hypothesis of Theorem 3.1 is singular, then $\rho(\mathcal{L}(\alpha, P))=1$. Note also that such an $A$ is singular if and only if 0 is a reducing eigenvalue. This happens if and only if $\mathcal{H}(A)$ and $\mathcal{S}(A)$ have a common null vector.

Note that in general, a matrix may have an eigenvalue of the form $i \xi$ which is not a reducing eigenvalue. However, this cannot happen for matrices $A$ such that $\mathcal{H}(A)$ is positive semidefinite.

For matrices $A$ such that $\mathcal{H}(A)$ is positive semidefinite, we need to determine whether it has no (reducing) eigenvalue of the form $i \xi$ with $\xi \in \mathbf{R}$. The next proposition gives some information along this direction.

Proposition 3.3. Suppose that $A \in \mathbf{C}^{n \times n}$ satisfies the hypothesis of Theorem 3.1. Then the following statements are equivalent:

(a) $A$ does not have a (reducing) eigenvalue of the form $i \xi$ with $\xi \in \mathbf{R}$.

(b) The null space of $\mathcal{H}(A)$ does not contain an eigenvector of $\mathcal{S}(A)$.

(c) If $v$ is an eigenvector of $\mathcal{S}(A)$, then $v^{*} \mathcal{H}(A) v>0$.

(d) Let $V$ be unitary such that $V^{*} \mathcal{H}(A) V=H_{1} \oplus 0_{\ell}$ where $H_{1}$ is nonsingular, and let

$$
V^{*} \mathcal{S}(A) V=\left(\begin{array}{cc}
S_{1} & E \\
-E^{*} & S_{2}
\end{array}\right)
$$

Then the null space of $E$ does not contain an eigenvector of $S_{2}$.

Proof. The equivalence of (a), (b), and (c) are straightforward. Now we consider (d). Suppose that $V$ is unitary such that $V^{*} \mathcal{H}(A) V=H_{1} \oplus 0_{\ell}$, where $H_{1}$ is nonsingular, and

$$
V^{*} \mathcal{S}(A) V=\left(\begin{array}{cc}
S_{1} & E \\
-E^{*} & S_{2}
\end{array}\right)
$$

Then a vector in the null space of $\mathcal{H}(A)$ must be of the form

$$
V\left(\begin{array}{l}
0 \\
x
\end{array}\right) \text {, with } x \in \mathbf{C}^{\ell} .
$$

Furthermore, it is an eigenvector of $\mathcal{S}(A)$ corresponding to the eigenvalue $i \xi$ with $\xi \in \mathbf{R}$ if and only if $E x=0$ and $S_{2} x=i \xi x$. Thus, (a) and (d) are equivalent.

From Theorem 3.1 and Proposition 3.3 , one can easily deduce the convergence results on the HSS iteration method for positive definite matrices in [3] and on the PHSS iteration methods for special positive semidefinite saddle-point matrices (1.2) in 2, 4, 11]. In the former case, it is clear that no eigenvalue has the form $i \xi$ with $\xi \in \mathbf{R}$. In the lattter case, under the assumption that $E$ has full column rank, condition (d) in Proposition 3.3 cannot hold.

The following example shows that even if $A$ is in two-by-two block form with

$$
\mathcal{H}(A)=\left(\begin{array}{cc}
H_{1} & 0 \\
0 & H_{2}
\end{array}\right) \quad \text { and } \quad \mathcal{S}(A)=\left(\begin{array}{cc}
S_{1} & E \\
-E^{*} & S_{2}
\end{array}\right)
$$

the relation between the null spaces of the matrices $H_{1}, H_{2}, E$, and $E^{*}$ may not be too useful in determining whether $\rho(\mathcal{L}(\alpha, P))<1$; see [11].

Example 3.4. Suppose that $A \in \mathbf{C}^{n \times n}$ is such that

$$
\mathcal{H}(A)=\left(\begin{array}{cc}
H_{0} & 0 \\
0 & H_{0}
\end{array}\right) \quad \text { with } \quad H_{0}=\left(\begin{array}{ll}
1 & 1 \\
1 & 1
\end{array}\right) .
$$


(a) If

$$
\mathcal{S}(A)=\left(\begin{array}{cc}
0 & E \\
-E^{*} & 0
\end{array}\right) \quad \text { with } \quad E=\left(\begin{array}{ll}
1 & 0 \\
0 & 1
\end{array}\right)
$$

then $A$ has eigenvalues $\pm i$. So, $\rho(\mathcal{L}(\alpha, I))=1$. However, it holds that $\operatorname{null}\left(H_{0}\right) \cap \operatorname{null}(E)=\{0\}$.

(b) If

$$
\mathcal{S}(A)=\left(\begin{array}{cc}
0 & E \\
-E^{*} & 0
\end{array}\right) \quad \text { with } \quad E=\left(\begin{array}{ll}
1 & 0 \\
0 & 0
\end{array}\right)
$$

then $A$ has no eigenvalue of the form $i \xi$ with $\xi \in \mathbf{R}$. So, $\rho(\mathcal{L}(\alpha, I))<1$. However, it holds that $\operatorname{null}\left(H_{0}\right) \cap \operatorname{null}(E)=\{0\}$.

(c) If

$$
\mathcal{S}(A)=\left(\begin{array}{cc}
S_{0} & 0 \\
0 & S_{0}
\end{array}\right) \quad \text { with } \quad S_{0}=\left(\begin{array}{cc}
0 & 1 \\
-1 & 0
\end{array}\right)
$$

then $A$ has no eigenvalue of the form $i \xi$ with $\xi \in \mathbf{R}$. So, $\rho(\mathcal{L}(\alpha, I))<1$. However, it holds that $\operatorname{null}\left(H_{0}\right) \cap \operatorname{null}(E)=\{0\}$.

(d) If

$$
\mathcal{S}(A)=\left(\begin{array}{cc}
S_{0} & E \\
-E^{*} & S_{0}
\end{array}\right) \quad \text { with } \quad S_{0}=\left(\begin{array}{cc}
0 & 1 \\
-1 & 0
\end{array}\right)
$$

so that $\|E\|$ is small, then $A$ has eigenvalues close to 1 . Thus, $\rho(\mathcal{L}(\alpha, I))=$ 1. However, we can choose $E$ such that either $\operatorname{null}\left(H_{0}\right) \cap \operatorname{null}(E)=\{0\}$ or $\operatorname{null}\left(H_{0}\right) \cap \operatorname{null}(E) \neq\{0\}$ holds.

If $\mathcal{H}(A)$ or $\mathcal{S}(A)$ is in diagonal block form

$$
B_{1} \oplus B_{2} \oplus \cdots \oplus B_{\ell}, \quad \text { with } \quad B_{j} \in \mathbf{C}^{n_{j} \times n_{j}}(j=1,2, \ldots, \ell),
$$

then one can consider

$$
P=\alpha_{1} I_{n_{1}} \oplus \alpha_{2} I_{n_{2}} \oplus \cdots \oplus \alpha_{\ell} I_{n_{\ell}}, \quad \text { with } \quad \alpha_{j}>0(j=1,2, \ldots, \ell) .
$$

As long as $\widehat{A}:=P^{-1 / 2} A P^{-1 / 2}$ does not have an eigenvalue of the form $i \xi$ with $\xi \in \mathbf{R}$, then the iteration matrix $\mathcal{L}(\alpha, P)$ of the PHSS iteration method has spectral radius less than one, i.e., the PHSS iteration scheme converges. In particular, when $\ell=1$, this conclusion recovers the convergence theorem established in 11 .

We remark that one may relax the condition that $\mathcal{H}(A)=\frac{1}{2}\left(A+A^{*}\right)$ is positive semidefinite. In fact, if there exists a $\theta \in[0,2 \pi)$ such that

$$
\mathcal{H}\left(e^{i \theta} A\right)=\frac{1}{2}\left(e^{i \theta} A+e^{-i \theta} A^{*}\right)
$$

is positive semidefinite, then one can apply Theorem 3.1 to $e^{i \theta} A$. This latter condition is equivalent to the requirement that the numerical range of $A$, defined by

$$
\mathcal{W}(A):=\left\{v^{*} A v: v \in \mathbf{C}^{n}, v^{*} v=1\right\},
$$

lie on a closed half plane defined by a line passing through the origin. In particular, if $\theta=\frac{\pi}{2}$ is such that $\mathcal{H}\left(e^{i \theta} A\right)$ is positive semidefinite, then the PHSS iteration method that results from interchanging the Hermitian matrix $\mathcal{H}(A)$ and the skewHermitian matrix $\mathcal{S}(A)$ may still converge. Note that in this PHSS iteration method the right-hand side vector $b$ is replaced by $i b$, correspondingly. 


\section{Applications}

We consider the non-Hermitian system of linear equations (1.1) whose coefficient matrix $A \in \mathbf{C}^{n \times n}$ is in the block tridiagonal form, i.e.,

$$
A x \equiv\left(\begin{array}{ccccc}
A_{1} & E_{1} & & & \\
-E_{1}^{*} & A_{2} & E_{2} & & \\
& \ddots & \ddots & \ddots & \\
& & -E_{\ell-2}^{*} & A_{\ell-1} & E_{\ell-1} \\
& & & -E_{\ell-1}^{*} & C
\end{array}\right)\left(\begin{array}{c}
x_{1} \\
x_{2} \\
\vdots \\
x_{\ell-1} \\
x_{\ell}
\end{array}\right)=\left(\begin{array}{c}
b_{1} \\
b_{2} \\
\vdots \\
b_{\ell-1} \\
b_{\ell}
\end{array}\right) \equiv b,
$$

where $A_{j} \in \mathbf{C}^{n_{j} \times n_{j}}(j=1,2, \ldots, \ell-1)$ are non-Hermitian matrices, $E_{j} \in \mathbf{C}^{n_{j} \times n_{j+1}}$ $(j=1,2, \ldots, \ell-1), C \in \mathbf{C}^{n_{\ell} \times n_{\ell}}$ is a Hermitian matrix, $x_{j}, b_{j} \in \mathbf{C}^{n_{j}}$, and $n_{j}(j=$ $1,2, \ldots, \ell)$ are positive integers satisfying $n_{1} \geq n_{2} \geq \cdots \geq n_{\ell}$ and $\sum_{j=1}^{\ell} n_{j}=n$.

The block tridiagonal systems of linear equations may arise from many applications, e.g., the remaining (linearized) Euler-Lagrange equations 28, 29] and a coupled DEM-FEM formulation combined with Lagrange multipliers in the imperious porous material with an incompressible pore fluid 23 .

In particular, when $\ell=2$, the system of linear equations (4.1) reduces to the generalized saddle-point problem (1.3) . As is known, saddle-point problems correspond to the Kuhn-Tucker conditions for linearly constrained quadratic programming problems, which typically result from mixed or hybrid finite element approximations of second-order elliptic problems, elasticity problems, or the Stokes equations (see, e.g., Brezzi and Fortin [13]) and from Lagrange multiplier methods (see, e.g., Fortin and Glowinski [17]). A number of structured preconditioners 15, 16, 25, 11, 10 and iterative methods [14, 22, 4, 2, 9, have been studied in the literature for these problems. See also [27, 21, 20, 26, 19, 18, 6, 8] and the references therein.

In this section we consider the block tridiagonal systems of linear equations satisfying all of the following assumptions:

- $A_{1}$ is positive definite, i.e., $\mathcal{H}\left(A_{1}\right)$ is Hermitian positive definite;

- $A_{j}(j=2,3, \ldots, \ell-1)$ are positive semidefinite, i.e., $\mathcal{H}\left(A_{j}\right)(j=2,3, \ldots, \ell-$ 1) are Hermitian positive semidefinite;

- $E_{j}(j=1,2, \ldots, \ell-2)$ have full column rank;

- $C$ is Hermitian positive semidefinite;

- $\operatorname{null}(C) \cap \operatorname{null}\left(E_{\ell-1}\right)=\{0\}$.

As shown below, these assumptions guarantee existence and uniqueness of the solution.

Proposition 4.1. Let $A \in \mathbf{C}^{n \times n}$ be the coefficient matrix of the system of linear equations (4.1). Assume that $A_{1}$ is positive definite, $A_{j}(j=2,3, \ldots, \ell-1)$ are positive semidefinite, $E_{j}(j=1,2, \ldots, \ell-2)$ have full column rank, $C$ is Hermitian positive semidefinite, and $\operatorname{null}(C) \cap \operatorname{null}\left(E_{\ell-1}\right)=\{0\}$. Then $A$ is nonsingular.

Proof. Let $x=\left(x_{1}^{*}, x_{2}^{*}, \ldots, x_{\ell}^{*}\right)^{*} \in \mathbf{C}^{n}$ be such that $A x=0$, where $x_{j} \in \mathbf{C}^{n_{j}}$ for $j=1,2, \ldots, \ell$. Then

$$
\left\{\begin{array}{l}
A_{1} x_{1}+E_{1} x_{2}=0, \\
-E_{j-1}^{*} x_{j-1}+A_{j} x_{j}+E_{j} x_{j+1}=0, \quad j=2,3, \ldots, \ell-1, \\
-E_{\ell-1}^{*} x_{\ell-1}+C_{\ell} x_{\ell}=0 .
\end{array}\right.
$$


Because $A x=0$ implies both $x^{*} A x=0$ and $x^{*} A^{*} x=0$, we know that $x^{*} \mathcal{H}(A) x=$ 0 . As $\mathcal{H}\left(A_{j}\right)(j=1,2, \ldots, \ell-1)$ and $C$ are Hermitian positive semidefinite, $\mathcal{H}(A)$ is Hermitian positive semidefinite, too. Hence, $x \in \operatorname{null}(\mathcal{H}(A))$, or equivalently,

$$
C x_{\ell}=0 \quad \text { and } \mathcal{H}\left(A_{j}\right) x_{j}=0 \quad \text { for } j=1,2, \ldots, \ell-1 .
$$

The system of linear equations (4.2) then reduces to the following:

$$
\left\{\begin{array}{l}
\mathcal{S}\left(A_{1}\right) x_{1}+E_{1} x_{2}=0, \\
-E_{j-1}^{*} x_{j-1}+\mathcal{S}\left(A_{j}\right) x_{j}+E_{j} x_{j+1}=0, \quad j=2,3, \ldots, \ell-1, \\
-E_{\ell-1}^{*} x_{\ell-1}=0 .
\end{array}\right.
$$

Since $\mathcal{H}\left(A_{1}\right)$ is Hermitian positive definite, we see that $x_{1}=0$. Based on (4.3) and the assumption that $E_{j}$ has full column rank for $j=1,2, \ldots, \ell-2$, we can successively obtain $x_{j}=0$ for $j=1,2, \ldots, \ell-1$. Thereby, (4.3) can be further reduced to

$$
E_{\ell-1} x_{\ell}=0 .
$$

Since $C x_{\ell}=0$, we conclude that $x_{\ell} \in \operatorname{null}(C) \cap \operatorname{null}\left(E_{\ell-1}\right)$, which is $\{0\}$ by our assumption. Hence, $x_{\ell}=0$. Therefore, the only solution for $A x=0$ is the trivial solution, and $A$ is nonsingular.

For the PHSS iteration method described in Section 2, if we first specifically take the Hermitian positive definite matrix $P \in \mathbf{C}^{n \times n}$ to be of block diagonal form, i.e.,

$$
P=\operatorname{Diag}\left(\frac{\alpha_{1}}{\alpha} P_{1}, \frac{\alpha_{2}}{\alpha} P_{2}, \ldots, \frac{\alpha_{\ell}}{\alpha} P_{\ell}\right)
$$

with

$$
\alpha_{j}>0 \text { and } P_{j} \in \mathbf{C}^{n_{j} \times n_{j}} \text { Hermitian positive definite, } j=1,2, \ldots, \ell,
$$

and then directly apply it to the block tridiagonal system of linear equations (4.1), the following iteration scheme, called the block preconditioned Hermitian and skewHermitian splitting (BPHSS) iteration method, can be obtained immediately.

The BPHSS iteration method. Let $P_{j} \in \mathbf{C}^{n_{j} \times n_{j}}(j=1,2, \ldots, \ell)$ be prescribed Hermitian positive definite matrices and let $\alpha_{j}(j=1,2, \ldots, \ell)$ be given positive constants. Given an initial guess

$$
x^{(0)}=\left(x_{1}^{(0)^{*}}, x_{2}^{(0)^{*}}, \ldots, x_{\ell}^{(0)^{*}}\right)^{*} \in \mathbf{C}^{n} \quad \text { with } \quad x_{j}^{(0)} \in \mathbf{C}^{n_{j} \times n_{j}},
$$

compute

$$
x^{(k)}=\left(x_{1}^{(k)^{*}}, x_{2}^{(k)^{*}}, \ldots, x_{\ell}^{(k)^{*}}\right)^{*} \in \mathbf{C}^{n} \quad \text { with } \quad x_{j}^{(k)} \in \mathbf{C}^{n_{j} \times n_{j}}
$$

for $k=0,1,2, \ldots$ using the following iteration scheme until $\left\{x^{(k)}\right\}$ satisfies the stopping criterion:

- Solve $x_{j}^{\left(k+\frac{1}{2}\right)}(j=1,2, \ldots, \ell)$ successively from the subsystems of linear equations

$$
\begin{aligned}
& \left(\alpha_{j} P_{j}+\mathcal{H}\left(A_{j}\right)\right) x_{j}^{\left(k+\frac{1}{2}\right)}=\left(\alpha_{j} P_{j}-\mathcal{S}\left(A_{j}\right)\right) x_{j}^{(k)}+E_{j-1}^{*} x_{j-1}^{(k)}-E_{j+1} x_{j+1}^{(k)}+b_{j}, \\
& j=1,2, \ldots, \ell \text {. }
\end{aligned}
$$


- Solve $x_{j}^{(k+1)}(j=1,2, \ldots, \ell)$ from the system of linear equations

$$
\begin{gathered}
\left(\alpha_{j} P_{j}+\mathcal{S}\left(A_{j}\right)\right) x_{j}^{(k+1)}-E_{j-1}^{*} x_{j-1}^{(k+1)}+E_{j+1} x_{j+1}^{(k+1)}=\left(\alpha_{j} P_{j}-\mathcal{H}\left(A_{j}\right)\right) x_{j}^{\left(k+\frac{1}{2}\right)}+b_{j}, \\
j=1,2, \ldots, \ell .
\end{gathered}
$$

Here, we have stipulated that $x_{0}^{(k)}=x_{0}^{(k+1)}=0$ and $x_{\ell+1}^{(k)}=x_{\ell+1}^{(k+1)}=0$.

Note that in the BPHSS iteration method, for each fixed iteration index $k$, the block vectors $x_{j}^{\left(k+\frac{1}{2}\right)}(j=1,2, \ldots, \ell)$ can be computed independently and, hence, the vector $x^{\left(k+\frac{1}{2}\right)}$ can be easily obtained on a multiprocessor system. Comparatively, the block vectors $x_{j}^{(k+1)}(j=1,2, \ldots, \ell)$ are more dependent, which may cause difficulty in solving the second-half iterate $x^{(k+1)}$ in parallel. However, there are efficient direct and iterative methods for solving this special class of block tridiagonal systems of linear equations; see [24, 21, 5, 1, 7]. Therefore, the BPHSS iteration method can be easily and effectively implemented in parallel on a multiprocessor system.

In addition, in actual computing it may be beneficial in solving the second-half iterate $x^{(k+1)}$ if we first execute block reordering for the system of linear equations (4.1), although this does not change the subsystem of linear equations defining the first-half iterate $x^{\left(k+\frac{1}{2}\right)}$.

The following theorem describes the convergence property of the BPHSS iteration method.

Theorem 4.2. Let all the conditions of Proposition 4.1 be satisfied. Then the $B P H S S$ iteration scheme is unconditionally convergent; that is, the spectral radius of its iteration matrix $\mathcal{L}\left(\alpha_{1}, \alpha_{2}, \ldots, \alpha_{\ell} ; P\right)$ satisfies

$$
\rho\left(\mathcal{L}\left(\alpha_{1}, \alpha_{2}, \ldots, \alpha_{\ell} ; P\right)\right)<1, \quad \text { for all } \alpha_{1}, \alpha_{2}, \ldots, \alpha_{\ell}>0 .
$$

Proof. Without loss of generality, we only need to consider the case that $P=I$; otherwise, we can turn to the preconditioned linear system (2.1) instead.

To prove the unconditional convergence of the BPHSS iteration method, according to Theorem 3.1 we only need to show that the null space of $\mathcal{H}(A)$ does not contain an eigenvector of $\mathcal{S}(A)$. In fact, if there exists a nonzero vector $x=\left(x_{1}^{*}, x_{2}^{*}, \ldots, x_{\ell}^{*}\right)^{*} \in \mathbf{C}^{n}$, with $x_{j} \in \mathbf{C}^{n_{j}}(j=1,2, \ldots, \ell)$, such that $\mathcal{H}(A) x=0$ and $\mathcal{S}(A) x=i \xi x$ hold for some $\xi \in \mathbf{R}$, i.e.,

$$
C x_{\ell}=0, \quad \mathcal{H}\left(A_{j}\right) x_{j}=0 \quad \text { for } j=1,2, \ldots, \ell-1,
$$

and

$$
\left\{\begin{array}{l}
\mathcal{S}\left(A_{1}\right) x_{1}+E_{1} x_{2}=i \xi x_{1}, \\
-E_{j-1}^{*} x_{j-1}+\mathcal{S}\left(A_{j}\right) x_{j}+E_{j} x_{j+1}=i \xi x_{j} \quad \text { for } j=2,3, \ldots, \ell-1, \\
-E_{\ell-1}^{*} x_{\ell-1}=i \xi x_{\ell},
\end{array}\right.
$$

then from the Hermitian positive definiteness of the matrix $\mathcal{H}\left(A_{1}\right)$ we know that $x_{1}=0$. It then follows from the full-rank assumption of the matrices $E_{j}(j=$ $1,2, \ldots, \ell-2)$ that $x_{j}=0(j=1,2, \ldots, \ell-1)$. Thus, (4.4) can be further reduced to

$$
E_{\ell-1} x_{\ell}=0 \quad \text { and } \quad i \xi x_{\ell}=0 .
$$

Evidently, whether $\xi=0$ or not, we can obtain $x_{\ell}=0$ due to the assumption $\operatorname{null}(C) \cap \operatorname{null}\left(E_{\ell-1}\right)=\{0\}$. Therefore, $x=0$, a contradiction. 
By suitable reordering or redecomposition, the block tridiagonal system of linear equations (4.1) can be reformulated as one with a two-by-two block coefficient matrix, or in the form of saddle-point problems. But now the $(1,1)$-block of the newly obtained two-by-two block matrix is not positive definite, even though its $(2,2)$-block is Hermitian positive semidefinite and the overlapping set between the null spaces of its $(1,2)$-block and $(2,2)$-block is $\{0\}$. Therefore, Theorem 3.1 in 11 cannot guarantee the convergence of the BPHSS iteration sequence. However Theorem 4.2 shows that the BPHSS iteration method is convergent unconditionally to the exact solution of the block tridiagonal system of linear equations (4.1).

\section{ACKNOWLEDGMENTS}

Part of this work was done during the first author's visits to the Department of Mathematics of The College of William and Mary during May-June 2004, and to Shanghai Key Laboratory of Contemporary Applied Mathematics of Fudan University.

\section{REFERENCES}

[1] Z.-Z. Bai, A class of parallel decomposition-type relaxation methods for large sparse systems of linear equations, Linear Algebra Appl., 282(1998), 1-24. MR1648288 (99j:65077)

[2] Z.-Z. Bai and G.H. Golub, Generalized preconditioned Hermitian and skew-Hermitian splitting methods for saddle-point problems, Technical Report SCCM-04-07, Scientific Computing and Computational Mathematics Program, Department of Computer Science, Stanford University, 2004.

[3] Z.-Z. Bai, G.H. Golub and M.K. Ng, Hermitian and skew-Hermitian splitting methods for non-Hermitian positive definite linear systems, SIAM J. Matrix Anal. Appl., 24(2003), 603626. MR1972670(2004c:65023)

[4] Z.-Z. Bai, G.H. Golub and J.-Y. Pan, Preconditioned Hermitian and skew-Hermitian splitting methods for non-Hermitian positive semidefinite linear systems, Numer. Math., 98(2004), 132. MR2076052 (2005h:65041)

[5] Z.-Z. Bai and Y.-F. Su, On the convergence of a class of parallel decomposition-type relaxation methods, Appl. Math. Comput., 81:1(1997), 1-21. MR1417759 (97g:65097)

[6] Z.-Z. Bai and G.-Q. Li, Restrictively preconditioned conjugate gradient methods for systems of linear equations, IMA J. Numer. Anal., 23:4(2003), 561-580. MR2011340(2004i:65025)

[7] Z.-Z. Bai and R. Nabben, Some properties of the block matrices in the parallel decompositiontype relaxation methods, Appl. Numer. Math., 29:2(1999), 167-170. MR1666521(99k:65038)

[8] Z.-Z. Bai and M.K. Ng, On inexact preconditioners for nonsymmetric matrices, SIAM J. Sci. Comput., 26:5(2005), 1710-1724. MR2142592 (2006d:65026)

[9] Z.-Z. Bai, B.N. Parlett and Z.-Q. Wang, On generalized successive overrelaxation methods for augmented linear systems, Numer. Math., 102:1(2005), 1-38.

[10] Z.-Z. Bai and Z.-Q. Wang, Restrictive preconditioners for conjugate gradient methods for symmetric positive definite linear systems, J. Comput. Appl. Math., 187(2006), 202-226. MR2185669

[11] M. Benzi and G.H. Golub, A preconditioner for generalized saddle point problems, SIAM J. Matrix. Anal. Appl., 26(2004), 20-41. MR2112850 (2005j:65026)

[12] M. Bertaccini, G.H. Golub, S. Serra-Capizzano and C.T. Possio, Preconditioned HSS methods for the solution of non-Hermitian positive definite linear systems and applications to the discrete convection-diffusion equation, Numer. Math., 99:3(2005), 441-484. MR.2117735

[13] F. Brezzi and M. Fortin, Mixed and Hybrid Finite Element Methods, Springer-Verlag, New York and London, 1991. MR1115205 (92d:65187)

[14] S.C. Eisenstat, H.C. Elman and M.H. Schultz, Variational iterative methods for nonsymmetric systems of linear equations, SIAM J. Numer. Anal., 20(1983), 345-357. MR0694523 (84h:65030) 
[15] H.C. Elman and M.H. Schultz, Preconditioning by fast direct methods for nonself-adjoint nonseparable elliptic equations, SIAM J. Numer. Anal., 23(1986), 44-57. MR0821905 (87g:65124)

[16] H.C. Elman, D.J. Silvester and A.J. Wathen, Performance and analysis of saddle point preconditioners for the discrete steady-state Navier-Stokes equations, Numer. Math., 90(2002), 665-688. MR1888834 (2002m:76071)

[17] M. Fortin and R. Glowinski, Augmented Lagrangian Methods, Applications to the Numerical Solution of Boundary Value Problems, North-Holland, Amsterdam, 1983. MR 0724072 (85a:49004)

[18] G.H. Golub and C. Greif, On solving block-structured indefinite linear systems, SIAM J. Sci. Comput., 24(2003), 2076-2092. MR2005622 (2004h:65030)

[19] G.H. Golub, C. Greif and J.M. Varah, Block orderings for tensor-product grids in two and three dimensions, Numer. Algorithms, 30(2002), 93-111. MR1917293 (2003f:65182)

[20] G.H. Golub and D. Vanderstraeten, On the preconditioning of matrices with skew-symmetric splittings, Numer. Algorithms, 25(2000), 223-239. MR1827156(2002e:65067)

[21] G.H. Golub and C.F. Van Loan, Matrix Computations, 3rd Edition, The Johns Hopkins University Press, Baltimore, 1996. MR1417720 (97g:65006)

[22] G.H. Golub and A.J. Wathen, An iteration for indefinite systems and its application to the Navier-Stokes equations, SIAM J. Sci. Comput., 19(1998), 530-539. MR.1618828 (99d:65107)

[23] H. Modaressi and P. Aubert, A diffuse element-finite element technique for transient coupled analysis, Intern. J. Numer. Methods Engrg., 39(1996), 3809-3838. MR.1417791 (97i:76077)

[24] J.J. Modi, Parallel Algorithms and Matrix Computation, In Oxford Applied Mathematics and Computing Science Series, J.N. Buxton, R.F. Churchhouse and A.B. Tayler eds., Clarendon Press, Oxford, 1988.

[25] M.F. Murphy, G.H. Golub and A.J. Wathen, A note on preconditioning for indefinite linear systems, SIAM J. Sci. Comput., 21(2000), 1969-1972. MR.1762024 (2001a:65055)

[26] C.-L. Wang and Z.-Z. Bai, Sufficient conditions for the convergent splittings of nonHermitian positive definite matrices, Linear Algebra Appl., 330(2001), 215-218. MR 1826657 (2002a:65059)

[27] A.J. Wathen and D.J. Silvester, Fast iterative solution of stabilized Stokes systems. Part I: Using simple diagonal preconditioners, SIAM J. Numer. Anal., 30(1993), 630-649. MR1220644 (94a:65060)

[28] S.L. Weissman, High-accuracy low-order three-dimensional brick elements, Intern. J. Numer. Methods Engrg., 39(1996), 2337-2361. MR.1399024 (97b:73094)

[29] J.-X. Zhao, W.-G. Wang and W.-Q. Ren, Stability of the matrix factorization for solving block tridiagonal symmetric indefinite linear systems, BIT, 44(2004), 181-188. MR2057369 (2005a:65030)

Department of Mathematics, Fudan University, Shanghai 200433, People's Republic of China, and State Key Laboratory of Scientific/Engineering Computing, Institute of Computational Mathematics and Scientific/Engineering Computing, Academy of Mathematics and Systems Science, Chinese Academy of Sciences, P.O. Box 2719, Beijing 100080, People's Republic of China

E-mail address: bzz@lsec.cc.ac.cn

Scientific Computing and Computational Mathematics Program, Department of Computer Science, Stanford University, Stanford, California 94305-9025

E-mail address: golub@sccm.stanford.edu

Department of Mathematics, The College of William \& Mary, P.O. Box 8795, WiLLiAMSBURG, Virginia 23187-8795

E-mail address: ckli@math.wm.edu 